\section{A randomized controlled trial of zinc supplementation in the treatment of acute respiratory tract infection in Thai children}

\author{
Sanguansak Rerksuppaphol,1 \\ Lakkana Rerksuppaphol² \\ 1Department of Pediatrics and \\ 2Department of Preventive Medicine, \\ Faculty of Medicine, Srinakharinwirot \\ University, Thailand
}

\section{Abstract \\ Acute Lower Respiratory Tract} Infections (ALRI) are one of the most common causes of morbidity and mortality in young children. Zinc supplementation has been shown to have a preventive effect against respiratory infections, but little evidence is available on its effect on the treatment of ALRI. This study examined the effect of zinc supplementation on the treatment outcome in children that were hospitalized with ALRI. A randomized, doubleblinded, placebo-controlled trial was conducted on 64 hospitalized children with ALRI, who were aged between 2 and 60 months. Children were randomly allocated to receive zinc ( $30 \mathrm{mg}$ elemental zinc/day) or placebo. The primary outcome was the time to the cessation of ALRI, while the secondary outcomes were the length of the stay in hospital and the individual features of the disease. The study found that ALRI cessation was faster in children who received zinc supplementation (median (IQR): 3 (2-4) days and 4 (3-5) days, respectively; $\mathrm{P}=0.008$ ), and that their hospital stay was shorter (mean (SD): 3.8 (1.3) days and 6.1 (3.2) days, respectively; $\mathrm{P}<0.001)$ than the placebo group. Zinc supplementation was well-tolerated, and no adverse events were reported. In conclusion, zinc supplementation reduced the number of days of ALRI in Thai children, as well as their stay in hospital.

\section{Introduction}

An Acute Lower Respiratory Tract Infection (ALRI), which is defined as any acute infection involving the lower part of the respiratory system, from the trachea to the lung parenchyma, is a leading cause of morbidity and mortality in young children, especially in developing countries. It is estimated that approximately 120-156 million children suffer from ALRI globally per year and that around $7-13 \%$ of all ALRI cases may progress to become severe diseases that warrant hospitalization. ${ }^{1}$ The burden of ALRI is high, with around 1.4 million children dying from such infections, especially those living in lower- and middle-income countries. ${ }^{1}$ Estimates of the World Health Organization suggest that improvements in the density, diversity, and quality of complementary foods may reduce the risk of ALRI incidence or mortality. ${ }^{2}$

Zinc supplementation has been studied extensively in developing countries, where zinc deficiency is common, as this element is an essential micronutrient for many physiological functions. With regard to the immune response, zinc directly promotes an antiviral activity and the immune-related release of interferon, ${ }^{3}$ and regulates the host's immune response to infection, by enhancing the mucous membrane barriers, the leukocyte function, and the cytokine expression. 4 Zinc is essential for the immune system, and a zinc deficiency has been reported to be associated with the increased chance of several infections, including ALRI.5 Several studies have shown that children with ALRI, regardless of their nutritional status, have had significantly lower serum zinc levels than those in healthy control children. ${ }^{6-9}$ A meta-analysis of the randomized efficacy trials that have been conducted in developing countries has confirmed that routine zinc supplementation reduces the incidence of childhood ALRI, thus indicating the potential protective effect of zinc supplementation against this disease. ${ }^{10}$ However, evidence on zinc supplementation as an adjuvant therapy in children with ALRI is still controversial.5,1115 Three studies on Indian and Bangladeshi children have found that zinc supplementation, as an adjuvant therapy, is beneficial for ALRI and severe pneumonia, as it helps to shorten the duration of hospitalization, and it also reduces the duration of the fever and the risk of treatment failure. ${ }^{11-13}$ On the contrary, other studies have found that zinc supplementation in Indigenous Australian and Indian children showed no clinical benefits, nor did it shorten the duration of the hospital stay. $5,14,15$

The exact national prevalence of zinc deficiency in Thailand is currently unknown. It is estimated that $41.6 \%$ of the population are at risk of inadequate zinc intake. 16 Two studies in North-east Thailand showed that $25-57 \%$ of young infants and school-going children had a low serum zinc concentration. ${ }^{17,18}$ The high prevalence of zinc deficiency in developing countries can be attributed to the inadequate intake of zinc-rich foods, as well as the malabsorption of zinc, which binds to the dietary fiber
Correspondence: Sanguansak Rerksuppaphol, Department of Pediatrics, Faculty of Medicine, Srinakhariwirot University, 62 Mo 7, RangsitNakorn Nayok Rd., NakornNayok, 26120 Thailand.

Tel.: +66.817231766 -Fax: +66.37395275

E-mail: sanguansak_r@hotmail.com

Key words: Child; Dietary supplements; Respiratory tract infection; Zinc.

Acknowledgements: the authors would like to thank all the children and their parents who participated in the study and all the people who contributed to this research project in different ways. They also wish to thank Elisa Sala, $\mathrm{PhD}$ and Medical Writer, for her helpful contribution in editing the text.

Contributions: the authors contributed equally

Conflict of interest: the authors declare no potential conflict of interest.

Funding: This study was supported by grants from the Srinakharinwirot University, Thailand.

Received for publication: 27 November 2018 Revision received: 7 April 2019.

Accepted for publication: 8 April 2019

This work is licensed under a Creative Commons Attribution NonCommercial 4.0 License (CC BY-NC 4.0).

(C) Copyright S. Rerksuppaphol and

L. Rerksuppaphol, 2019

Licensee PAGEPress, Italy

Pediatric Reports 2019; 11:7954

and phytate of plant foods that are commonly consumed in such countries. Zinc occurs in various food products, but it is found in high concentrations in animal source foods and in low concentrations in tubers, refined cereals, fruits and vegetables. On the contrary, phytate, which is a strong chelator of minerals, including zinc, is often found in seeds, cereal gains, nuts and legumes. ${ }^{16}$

Although the prevalence of zinc deficiency is high in Thailand, there have been no previous studies on the effects of zinc supplementation on Thai children with ALRI. In this study, we aimed to evaluate the effect of zinc supplementation during the treatment of acute respiratory tract infections in Thai children.

\section{Materials and Methods}

\section{Study design and subject}

A randomized, double-blind, placebocontrolled trial was conducted on children 
aged between 2 and 60 months, who were clinically diagnosed with ALRI and who were admitted to the Pediatric Unit of the MSMC Srinakharinwirot University Hospital between June 2017 and January 2018. The decisions regarding their admission and the general management of ALRI were made by the emergency and attending physicians, respectively. The diagnosis of ALRI was made in the presence of 1) tachypnea (a respiratory rate of more than 50 per minute for children aged 2-12 months, and more than 40 per minute for children older than 12 months), and 2) either of at least one other observed sign of ALRI [lower chest wall in-drawing, abnormal sounds on pulmonary auscultation (i.e. bronchial breath sounds and/or crackles/crepitations), nasal flaring and fever, or one of the following danger signs (i.e. cyanosis, lethargy, irritability, inability to drink or convulsions)]. ${ }^{10}$ Children with a known history of chronic illnesses, such as chronic liver or renal diseases, congenital heart diseases, immune deficiency or malignancy, were excluded. Children who regularly took vitamins or minerals, or those who had an allergy to them, were also excluded from the study. The study was conducted in compliance with the principles of the Declaration of Helsinki. The protocol was approved by the Ethics Committee of the Srinakharinwirot University. Written informed consent was obtained before enrolment from the parents or legal guardians, and the children could withdraw from the study at any time. The zinc supplements and placebo were purchased from a manufacturing company. The company played no role in the conception and design of the study, nor how it was conducted, or in the analysis and interpretation of the data. This study was registered with the Thai Clinical Trials Registry (TCTR20171211002).

\section{Intervention}

After enrolment, the children were randomized to receive zinc supplementation or placebo by an independent person who was not involved in the study. The randomization list was generated by using a computer program (GraphPad QuickCals, La Jolla, CA, USA), in a 1:1 ratio and in a block size of 2. An independent person prepared the randomization schedule and oversaw the packaging and labeling process to ensure the blinding. All investigators, children, parents, and guardians were blinded throughout the study. The randomization codes were opened after the completion of the study.

The children in the treatment group received chelated zinc as zinc bis-glycinate
(15 mg elemental zinc) twice a day until their discharge from the hospital, or up to a maximum of 7 days. Children in the control group received an ORS-based placebo, with similar appearance and taste to zinc powder. Both the zinc and placebo were manufactured and supplied by Qualimed (Bangkok, Thailand) as a powder in an identical single-dose sachet. The contents of the sachet were dissolved in a glass of water before administration. The treatment of ALRI, as well as the observation and the discharge decisions, were made by the attending physicians, who were not involved in the implementation phase of the study.

\section{Data collection and monitoring}

Baseline data were collected by trained research staff before any trial-related medication was administered. The detailed medical history and clinical assessments were undertaken by the attending physicians. Physical examinations were assessed by the same physician at the baseline, and then twice daily, until discharge. The axillary temperature was measured by using a clinical glass thermometer; the respiratory rate and oxygen saturation were measured by pulse oximetry and were recorded by the nurses every 4 hours, until the patients were discharged from the hospital. A fever was defined as an axillary temperature of $37.5^{\circ} \mathrm{C}$, or above. The resolution of a fever was defined as the first time that the temperature dropped to within the normal level for two consecutive measurements.

The serum zinc levels were measured by flame atomic absorption spectrometry at the baseline, as well as after seven days of supplementation or before the discharge. The time of blood drawing and the fasting status of the children were recorded. The lower cut-off thresholds of serum zinc to define the zinc deficiency in children $<10$ years old are as follows: morning non-fasting $65 \mathrm{mg} / \mathrm{dL}$, or afternoon $57 \mathrm{mg} / \mathrm{dL} .{ }^{19}$ Open-ended questions were used to assess the side-effects in both groups. Compliance to the treatment was assessed by the sum of the drug intake.

\section{Outcome measures}

The primary outcome of the study was the time to the cessation of ALRI, which is defined as the period starting from enrolment to the disappearance of: 1) tachypnea; 2) chest in-drawing; 3) abnormal pulmonary auscultation; 4) hypoxemia $\left(\mathrm{SpO}_{2} \leq 93 \%\right.$ in room air); and 5) any dangerous signs. The secondary outcomes were the duration of the hospital stay and the clinical features of the disease.

\section{Statistical analysis}

Sample size estimation: To gain a statistical power of $80 \%$ and a two-sided Type I error of 0.01 , and to detect a $7 \%$ shorter time of resolution of ALRI in the treatment group, compared to the control group of 97 hours (SD. 7 hours), a sample size of 26 per group was required. ${ }^{12}$ Allowing for a dropout of $15 \%$, we planned to enroll 64 children (32 in each group).

The normal distribution of continuous data was assessed by using the Kolmogorov-Smirnov test. Normally distributed variables were descriptively presented as mean and standard deviations, whereas non-normally distributed variables were reported as median and interquartile range (IQR). The Pearson chi-square test, or the Fisher exact test, was used to compare the proportions between the groups. Normally distributed continuous variables were compared, using the Student's t-test, whereas non-normally distributed variables were compared, using the Mann-Whitney U test. The time to event (cessation of ALRI) was evaluated by the Kaplan-Meier survival analysis and compared with the log-rank test. The change in serum zinc levels from the baseline was assessed by a paired t-test and presented as a mean and a $95 \%$ confidence interval (CI). The statistical analysis was performed with the SPSS 23.0 software (SPSS. Inc, Chicago, IL). A P-value of lower than 0.05 was considered to be statistically significant.

\section{Results}

Seventy children who were eligible to participate in the study were recruited; of these, 64 were randomized to receive either zinc supplementation $(\mathrm{n}=32)$ or placebo $(n=32)$. All participants completed the study protocol. A flow chart of the study and enrollment is reported in Figure 1.

The baseline demographic characteristics and clinical features are summarized in Table 1. Approximately two-thirds (67.2\%) of the children were males, with a median age of 18.5 months (IQR 11.5-32.3 months). The median duration of illness before hospitalization was 2 days (IQR 1-3 days), with chest in-drawing, nasal flaring and a sore throat being the most common clinical features. On admission, the mean serum zinc concentrations did not differ significantly between the two groups $(75.0 \pm 21.1 \mathrm{mg} / \mathrm{dL}$ in the treatment group and $76.6 \pm 22.6 \mathrm{mg} / \mathrm{dL}$ in placebo; $\mathrm{P}=0.767$ ). Overall, the prevalence of zinc deficiency in this population was $34.4 \%$, and there was no statistical difference between the groups. 
At the end of the study, the serum zinc concentration in the treatment group was significantly higher than that in the control group $(113.5 \pm 29.9 \mathrm{mg} / \mathrm{dL}$ and $89.4 \pm 21.5$ $\mathrm{mg} / \mathrm{dL} ; \mathrm{P}<0.001)$. However, both groups had a significantly higher mean serum zinc concentration than the baseline: the mean gain was $38.6 \mathrm{mg} / \mathrm{dL}$ (95\% CI: 26.2 to 51.0 $\mathrm{mg} / \mathrm{dL}$ ) in the treatment group and 12.8 $\mathrm{mg} / \mathrm{dL}$ (95\% CI: 3.9 to $21.7 \mathrm{mg} / \mathrm{dL})$ in the placebo group.

All ALRI symptoms disappeared in a significantly shorter time in the treatment group, compared to the control group (3.0 days and 4.0 days, respectively; $\mathrm{P}=0.008$ ) (Table 2). Within 7 days of hospitalization, ALRI was not resolved in one child in the treatment group and 3 children in control group $(\mathrm{P}=0.613)$. The recovery rate was consistently higher in the treatment group, compared to the control group, during the entire duration of the study $(\mathrm{P}=0.046)$ (Figure 2). Chest in-drawing (1.5 versus 2.0 days; $\mathrm{P}=0.021$ ), tachypnea ( 8.0 versus 24.0 hours; $\mathrm{P}<0.001)$, fever (10.0 versus 41.6 hours; $\mathrm{P}<0.001)$ and the duration of hospitalization (3.8 versus 6.1 days; $\mathrm{P}<0.001$ ) were significantly reduced. The time to achieve normal oxygenation $\left(\mathrm{SpO}_{2}>94 \%\right.$ in room air), the disappearance of wheezing, and normal bilateral air entry did not differ between the groups.

No adverse effects were reported; all patients assumed the assigned medication, as per schedule, and their adherence to the treatment was good.

\section{Discussion}

This study indicated that zinc supplementation (15 mg elemental zinc twice daily) after hospital admission significantly shortened the recovery time and duration of the hospital stay of Thai children with ALRI. Of the ALRI symptoms, the duration of chest in-drawing, tachypnea and fever were improved by the zinc intake, whereas the time to restore normal oxygenation, the disappearance of a wheeze, and normal bilateral air entry did not significantly change with the treatment. Overall, zinc supplementation, as zinc bis-glycinate, was well-tolerated and safe.

Data on the potential of zinc supplementation, as an adjuvant therapy for ALRI in children, are scarce and controversial, as they present a wide spectrum of study designs, forms of zinc and outcome measurements. Our results are in accordance with the results of other RCT studies, in which zinc supplementation reduced the duration of the hospital stay and significantly improved the recovery from ALRI and severe pneumonia. ${ }^{11-13}$ Mahalanabis et al. ${ }^{12}$ showed benefits of zinc acetate supplementation $(20 \mathrm{mg} /$ day $)$ in Indian children with severe ALRI. They concluded that zinc sup-

plementation significantly reduced the duration of fever and improved the recovery rate of very ill patients; however, this clinical significance was only observed in boys.

Table 1. Baseline demographic characteristics and clinical features of participants.

\begin{tabular}{|c|c|c|c|}
\hline Variables & Zinc $(\mathrm{n}=32)$ & Placebo $(n=32)$ & $\mathbf{P}$ \\
\hline Male, n (\%) & $24(75.0)$ & $19(59.4)$ & 0.287 \\
\hline Age, months* & $19(10-32)$ & $17(13-34.5)$ & 0.936 \\
\hline $\begin{array}{l}\text { Age group, } \mathrm{n}(\%) \\
2-12 \text { months } \\
13-24 \text { months } \\
25-60 \text { months }\end{array}$ & $\begin{array}{c}9(28.1) \\
12(37.5) \\
11(34.4)\end{array}$ & $\begin{array}{c}7(21.9) \\
15(46.9) \\
10(31.3)\end{array}$ & 0.729 \\
\hline Weight, kg & $11.4(4.3)$ & $10.5(2.4)$ & 0.305 \\
\hline Height, cm & $82.2(14.7)$ & $80.1(11.4)$ & 0.524 \\
\hline Days of illness before admission* & $2(1-3)$ & $2(1-3)$ & 0.577 \\
\hline $\begin{array}{l}\text { Clinical features } \\
\text { Body temperature, }{ }^{\circ} \mathrm{C} \\
\text { Respiratory rate, breaths/min } \\
\text { Pulse, beats/min } \\
\text { Systolic blood pressure, mmHg } \\
\text { Diastolic blood pressure, mmHg } \\
\text { Oxygen saturation at room air, \% } \\
\text { Rhinorrhea, n (\%) } \\
\text { Sore throat, n (\%) } \\
\text { Diarrhea, n (\%) } \\
\text { Vomiting, n (\%) } \\
\text { Chest in-drawing, n (\%) } \\
\text { Nasal flaring, n (\%) } \\
\text { Wheezing, n (\%) } \\
\text { Crepitation, n (\%) } \\
\text { Hemoglobin, g/dL } \\
\text { Hemoglobin }<11 \mathrm{~g} / \mathrm{dL}, \mathrm{n} \mathrm{( \% )} \\
\text { Hematocrit, } \% \\
\text { Leucocyte count, } \times 10^{3} / \mathrm{mm}^{3} \\
\text { Neutrophil, } \% \\
\text { Platelets, } \times 103 / \mathrm{mm}^{3} \\
\end{array}$ & $\begin{array}{c}38.4(0.9) \\
51(10) \\
165(16) \\
108(9) \\
62(9) \\
95.1(2.4) \\
31(96.9) \\
29(90.0) \\
4(12.5) \\
7(21.9) \\
30(93.8) \\
29(90.6) \\
17(53.1) \\
23(71.9) \\
11.7(1.3) \\
10(31.3) \\
35.7(3.4) \\
13.5(4.3) \\
59.6(17.9) \\
385.4(108.4) \\
\end{array}$ & $\begin{array}{c}38.5(0.8) \\
49(6) \\
171(20) \\
107(11) \\
65(10) \\
94.6(3.6) \\
30(93.8) \\
29(90.0) \\
3(9.4) \\
9(28.1) \\
31(96.9) \\
30(93.8) \\
14(43.8) \\
22(68.8) \\
11.3(1.4) \\
14(43.8) \\
34.8(3.4) \\
15.1(7.4) \\
61.4(16.1) \\
406.9(127.3) \\
\end{array}$ & $\begin{array}{l}0.619 \\
0.312 \\
0.190 \\
0.725 \\
0.216 \\
0.759 \\
1.000 \\
1.000 \\
1.000 \\
0.774 \\
1.000 \\
1.000 \\
0.617 \\
1.000 \\
0.293 \\
0.439 \\
0.276 \\
0.300 \\
0.683 \\
0.469\end{array}$ \\
\hline
\end{tabular}

Data were presented as mean (standard deviation) unless otherwise indicated; *Presented as median (interquartile range).

Table 2. Outcomes of zinc supplementation compared with placebo for children with acute lower respiratory tract infection.

\begin{tabular}{|c|c|c|c|}
\hline Variables & Zinc $(\mathrm{n}=32)$ & Placebo $(n=32)$ & $P$ \\
\hline Time to cessation of ALRIa, days & $3.0(2.0-4.0)$ & $4.0(3.0-5.0)$ & 0.008 \\
\hline Time to disappearance of tachypneab, hours & $8.0(4.0-19.0)$ & $24.0(13.0-45.5)$ & $<0.001$ \\
\hline Time to disappearance of chest in-drawing, days & $1.5(1.0-2.0)$ & $2.0(1.0-3.0)$ & 0.021 \\
\hline $\begin{array}{l}\text { Time to disappearance of abnormal pulmonary } \\
\text { auscultation, days }\end{array}$ & $2.0(0-3.0)$ & $1.0(0-2.75)$ & 0.387 \\
\hline Time to achieve $\mathrm{SpO}_{2}>94 \%$ in room air, days & $0(0-1.0)$ & $0(0-2.0)$ & 0.405 \\
\hline Time to normal bilateral air entry, days & $2.5(2.0-3.0)$ & $3.0(2.0-5.0)$ & 0.151 \\
\hline Time to resolution of fever, hours & $10.0(4.0-27.0)$ & $41.6(17.0-72.0)$ & $<0.001$ \\
\hline Days in Hospital, days, mean (SD) & $3.8(1.3)$ & $6.1(3.2)$ & $<0.001$ \\
\hline $\begin{array}{l}\text { Zinc levels, } \mu \mathrm{g} / \mathrm{dL} \text {, mean (SD) } \\
\text { Baseline } \\
\text { End of study }\end{array}$ & $\begin{array}{c}75.0(21.1) \\
113.5(29.9) \mathrm{c}\end{array}$ & $\begin{array}{c}76.6(22.6) \\
89.4(21.5) c\end{array}$ & $\begin{array}{l}0.767 \\
<0.001\end{array}$ \\
\hline $\begin{array}{l}\text { Zinc deficiency, n (\%) } \\
\text { Baseline } \\
\text { End of study }\end{array}$ & $\begin{array}{c}10(31.3) \\
0(0)\end{array}$ & $\begin{array}{l}12(37.5) \\
4(12.5)\end{array}$ & $\begin{array}{l}0.793 \\
0.113\end{array}$ \\
\hline
\end{tabular}

Data were presented as median (interquartile range), unless otherwise indicated. aTime to cessation of ALRI: time interval from enrolment to absent of tachypnea, chest in-drawing, abnormal pulmonary auscultation, hypoxemia and any danger signs. bDisappearance of tachypnea: respiratory rate $<50 /$ minute in aged 2-12 months or $<40 /$ minute in children aged $>12$ months. cSignificant differences from the baseline within each group $(\mathrm{P}<0.001)$. 
The results of a study by Basnet et al.,13 in which zinc sulphate (10-20 mg/day) supplementation was used on Indian children with severe ALRI (pneumonia), showed a marginal reduction in the time to the cessation of the disease and in the risk of treatment failure. Brooks et al. ${ }^{11}$ showed that the zinc supplementation of $20 \mathrm{mg} /$ day, in the form of zinc acetate, reduced the duration of hospitalization and accelerated the recovery time from severe ALRI (pneumonia) in Bangladeshi children. A number of previous studies found no beneficial effects of zinc supplementation on ALRI in children. $5,14,15$ Bose et al. ${ }^{14}$ studied the efficacy of zinc sulphate $(20 \mathrm{mg} /$ day $)$ supplementation in Indian children with severe ALRI (pneumonia). The study failed to demonstrate the significant clinical benefits of the adjuvant therapy or the duration of hospitalization. Chang et al. 15 found that giving zinc gluconate supplementation $(20-40 \mathrm{mg} /$ day $)$ to indigenous Australian children had no clinical benefit for the recovery from the symptoms, or on the duration of hospitalization. Instead, they found that children who were given zinc supplements had an increased risk of readmission with ALRI. In a study by Bansal et al.,5 it was found that zinc gluconate supplementation $(20 \mathrm{mg} /$ day $)$ in Indian children with ALRI did not reduce the recovery time or the duration of hospitalization.

The difference in the outcomes of our findings and those reported by other authors can be attributed to the different forms and dosages of the zinc supplements used. The dosage of zinc in the present study was approximately 2-3 times the recommended daily allowance for the treatment of zinc deficiency. ${ }^{20}$ Inorganic zinc, such as zinc sulphate, has a low bio-availability and there is a higher incidence of gastrointestinal side effects. The experimental evidence demonstrates that zinc, as an organic complex, is absorbed better than zinc, as an inorganic complex.21 However, the bioavailability of various forms of organic zinc complexes varies. The present study used zinc bis-glycinate, which is a new chelate that is formed by two glycine molecules bound to a zinc cation and which results in a double heterocyclic ring compound. A previous study has shown that zinc bis-glycinate, as used in this study, is safe and has a higher bioavailability than other organic zinc. ${ }^{22}$ This 1:2 metal to ligand ratio limits the inhibition of metal absorption by dietary components and is not involved in oxidative reactions. ${ }^{23}$ The chemical form of zinc determines its bioavailability and, hence, the amount that is absorbed and assimilated from the intestines after digestion. Zinc is found as organic complexes in meat products, and as inorganic salts in plant foods. 24,25 However, no isotopic studies have been carried out to assess the bioavailability of these different forms of zinc. The chelation of zinc to amino acids is known to improve the bioavailability of zinc, as a result of its increased solubility, in comparison to the inorganic forms of zinc, such as zinc sulfate. ${ }^{26}$ Furthermore, the administration of amino acid chelates minimizes symptoms, such as nausea and vomiting, epigastric pain, abdominal cramps and diarrhea, which have sometimes been reported during zinc supplementation. ${ }^{27}$

The prevalence of low-serum zinc level in hospitalized Thai children with ALRI was around $1 / 3(34.4 \%)$. This finding is consistent with studies in other countries that found low serum zinc levels in ALRI

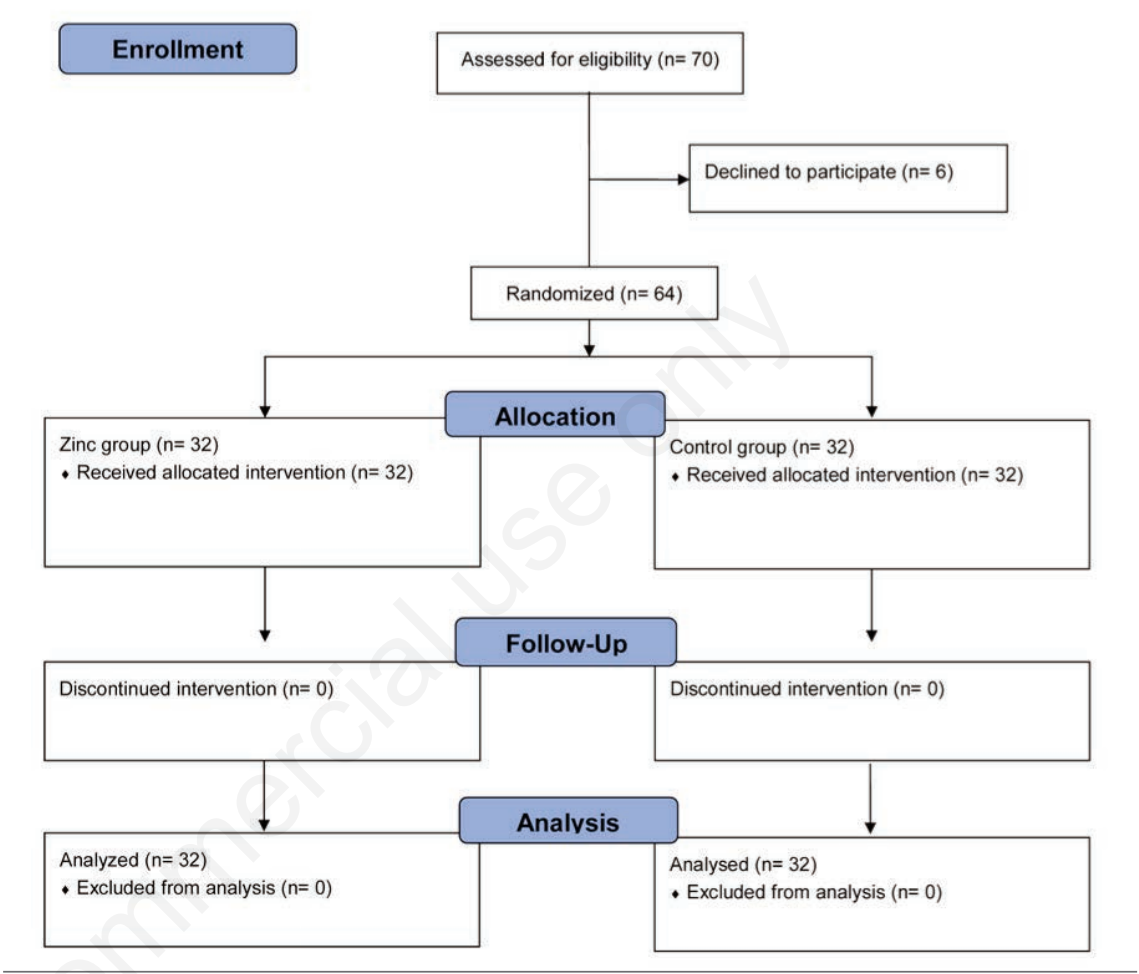

Figure 1. Study flowchart and enrolment.

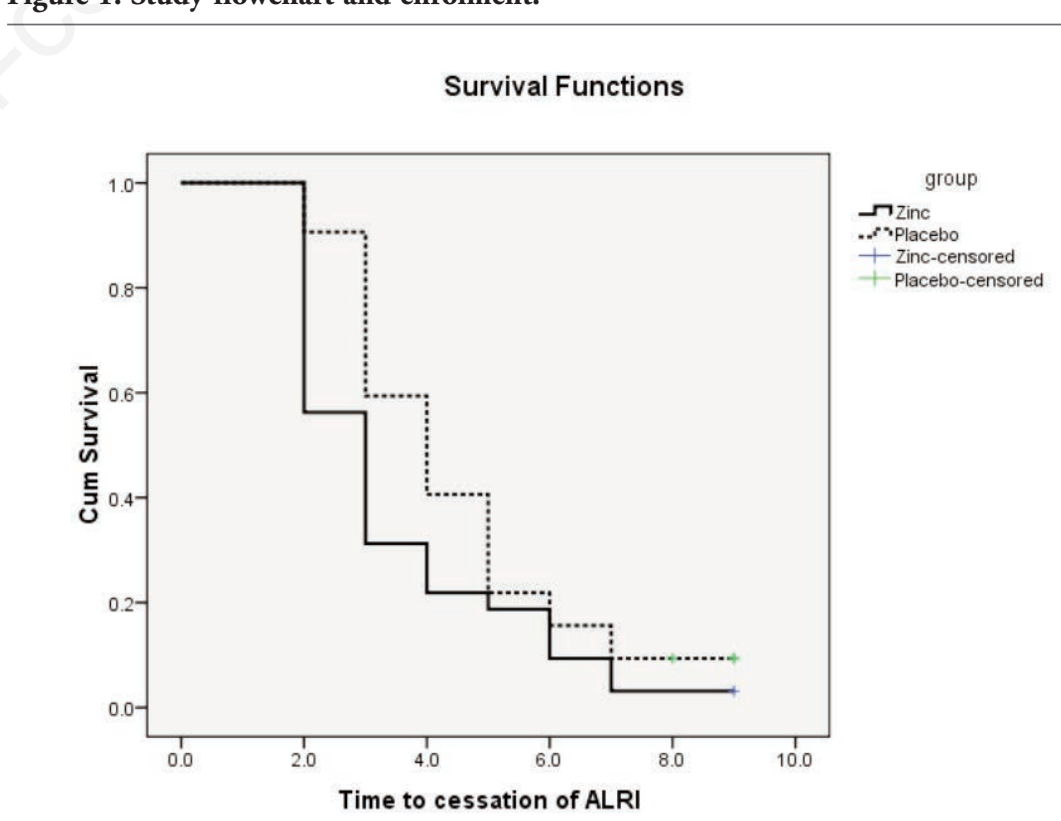

Figure 2 Kaplan-Meier survival curves for time to cessation of acute lower respiratory tract infection (ALRI). 
children.6-9 Low serum zinc levels were observed in Nigerian children that were hospitalized with ALRI, compared to the healthy controls. This difference may be attributed to a pre-existing state of zinc deficiency and its negative effects on the immune system of children. ${ }^{6}$ Nevertheless, even well-nourished Bangladeshi children with ALRI showed lower serum zinc levels, compared to the healthy controls, ${ }^{8}$ thus suggesting that zinc deficiency could impact their susceptibility to ALRI. Our study did not compare the zinc levels of ALRI patients with those of healthy children, since we were focused on defining the impact of zinc supplementation on the clinical outcome of ALRI; however, a further cohort study, which compares ALRI patients with normal control children, would be useful to specify the relationship between zinc deficiency and ALRI.

The mechanism by which zinc supplementation improves the symptoms of ALRI is unknown. Theoretically, zinc is essential for protein synthesis and cell growth, and it plays a critical role in maintaining the integrity of the immune system and respiratory cells during inflammation mucosal resistance. ${ }^{3,16,28}$ It activates several zincdependent enzymes and transcription factors, which are the gene expression of interleukin and interleukin receptors, as well as antioxidant and anti-inflammatory activities, thus promoting a direct anti-viral activity and an effect on the immune-mediated production of interferon. ${ }^{3,29}$ In respiratory infections, zinc deficiency may exacerbate the inflammation and cellular damage of the airway, and zinc supplementation in children may reduce the inflammation, lower the airway obstruction and shorten the duration of chest in-drawing, the high respiratory rate and hypoxia. ${ }^{11}$ Furthermore, zinc deficiency affects the antibody-mediated responses to infection, which are determined by avoiding the colonization and phagocytosis of encapsulated bacteria. 30 Based on this evidence, it is conceivable that increasing the zinc levels during ALRI contributes to the improved clinical outcomes. Thai children who have an estimated risk of zinc deficiency that is higher than $40 \%{ }^{16}$ may benefit from zinc supplementation, not only to increase the dietary uptake of micronutrients, but also to improve their recovery from illness. Although other studies will better clarify the role of zinc supplementation for the management of ALRI, our positive results have indicated that if zinc intake is administered from when a patient is admitted to hospital, it may be useful for accelerating the recovery of patients.

This study had some limitations. The treatment and the decisions on discharge were managed by attending physicians, whose attitudes were different and often confusing. However, to minimize the potential discrepancies, we considered the clinical signs, for example, the respiratory rate, the temperature, chest in-drawing, auscultation, and oxygen saturation, as the primary endpoints. Secondly, we did not assess and control the dietary intake of zinc during the study. Finally, we used a broad definition of ALRI and we did not classify the etiology by conducting blood cultures.

Further studies would be helpful to determine the optimal dosage required and to establish whether zinc supplementation could be useful to enhance the recovery and improve the clinical outcomes, even in children who are not affected by a zinc deficiency.

\section{Conclusions}

This randomized study indicated that zinc supplementation improved the recovery of Thai children with ALRI and reduced their hospital stay, compared to the placebo group. Our preliminary data has paved the way for further studies to establish the optimal dosage and the schedule for zinc supplementation, in an ALRI setting.

\section{References}

1. Nair H, Simoes EA, Rudan I, et al. Global and regional burden of hospital admissions for severe acute lower respiratory infections in young children in 2010: a systematic analysis. Lancet 2013;381:1380-90.

2. Roth DE, Caulfield LE, Ezzati M, et al. Acute lower respiratory infections in childhood: opportunities for reducing the global burden through nutritional interventions. Bull World Health Organ 2008;86:356-64.

3. Shankar AH, Prasad AS. Zinc and immune function: the biological basis of altered resistance to infection. Am J Clin Nutr 1998;68:447S-63S.

4. Bhatnagar S, Natchu UC. Zinc in child health and disease. Indian $\mathrm{J}$ Pediatr 2004;71:991-5.

5. Bansal A, Parmar VR, Basu S, et al. Zinc supplementation in severe acute lower respiratory tract infection in children: a triple-blind randomized placebo controlled trial. Indian J Pediatr 2011; 78:33-7.

6. Ibraheem RM, Johnson AB, Abdulkarim AA, et al. Serum zinc levels in hospitalized children with acute lower respiratory infections in the north-central region of Nigeria. Afr Health Sci 2014;14:136-42.

7. Shakur MS, Malek MA, Bano N, et al. Serum and hair zinc in severely malnourished Bangladeshi children associated with or without acute lower respiratory infection. Indian $\mathrm{J}$ Pediatr 2009;76:609-14.

8. Shakur MS, Malek MA, Bano N, et al. Zinc status in well nourished Bangladeshi children suffering from acute lower respiratory infection. Indian Pediatr 2004;41:478-81.

9. Kumar S, Awasthi S, Jain A, et al. Blood zinc levels in children hospitalized with severe pneumonia: a case control study. Indian Pediatr 2004;41:486-91.

10. Roth DE, Richard SA, Black RE. Zinc supplementation for the prevention of acute lower respiratory infection in children in developing countries: metaanalysis and meta-regression of randomized trials. Int $\mathrm{J}$ Epidemiol 2010;39:795-808.

11. Brooks WA, Yunus M, Santosham M, et al. Zinc for severe pneumonia in very young children: double-blind placebocontrolled trial. Lancet 2004;363:16838.

12. Mahalanabis D, Lahiri M, Paul D, et al. Randomized, double-blind, placebocontrolled clinical trial of the efficacy of treatment with zinc or vitamin A in infants and young children with severe acute lower respiratory infection. Am J Clin Nutr 2004;79:430-6.

13. Basnet S, Shrestha PS, Sharma A, et al. A randomized controlled trial of zinc as adjuvant therapy for severe pneumonia in young children. Pediatrics 2012;129:701-8.

14. Bose A, Coles CL, Gunavathi, et al. Efficacy of zinc in the treatment of severe pneumonia in hospitalized children $<2$ y old. Am J Clin Nutr 2006;83:1089-96; quiz 207.

15. Chang AB, Torzillo PJ, Boyce NC, et al. Zinc and vitamin A supplementation in Indigenous Australian children hospitalised with lower respiratory tract infection: a randomised controlled trial. Med J Aust 2006;184:107-12.

16. Brown KH, Rivera JA, Bhutta Z, et al. International Zinc Nutrition Consultative Group (IZiNCG) technical document \#1. Assessment of the risk of zinc deficiency in populations and options for its control. Food Nutr Bull 2004;25:S99-203.

17. Wasantwisut E, Winichagoon $P$, Chitchumroonchokchai $\mathrm{C}$, et al. Iron and zinc supplementation improved iron and zinc status, but not physical 
growth, of apparently healthy, breastfed infants in rural communities of northeast Thailand. J Nutr 2006;136:2405-11.

18. Thurlow RA, Winichagoon $P$, Pongcharoen $\mathrm{T}$, et al. Risk of zinc, iodine and other micronutrient deficiencies among school children in North East Thailand. Eur J Clin Nutr 2006;60:623-32.

19. Hotz C, Peerson JM, Brown KH. Suggested lower cutoffs of serum zinc concentrations for assessing zinc status: reanalysis of the second National Health and Nutrition Examination Survey data (1976-1980). Am J Clin Nutr 2003;78:756-64.

20. Saper RB, Rash R. Zinc: an essential micronutrient. Am Fam Physician 2009;79:768-72.

21. Yu Y, Lu L, Li SF, et al. Organic zinc absorption by the intestine of broilers in vivo. Br J Nutr 2017;117:1086-94.

22. Gandia P, Bour D, Maurette JM, et al. A bioavailability study comparing two oral formulations containing zinc ( $\mathrm{Zn}$ bis-glycinate vs. Zn gluconate) after a single administration to twelve healthy female volunteers. Int J Vitam Nutr Res 2007;77:243-8

23. Prasad AS. Discovery of human zinc deficiency: its impact on human health and disease. Adv Nutr 2013;4:176-90.

24. Gibson RS. The role of diet- and hostrelated factors in nutrient bioavailability and thus in nutrient-based dietary requirement estimates. Food Nutr Bull 2007;28:S77-100.

25. Solomons NW, Jacob RA, Pineda O, et al. Studies on the bioavailability of zinc in man. II. Absorption of zinc from organic and inorganic sources. J Lab Clin Med 1979;94:335-43.

26. Lonnerdal B. Dietary factors influenc- ing zinc absorption. J Nutr 2000;130:1378s-83s.

27. Brown MA, Thom JV, Orth GL, et al. Food Poisoning Involving Zinc Contamination. Arch Environ Health 1964;8:657-60.

28. Bao S, Knoell DL. Zinc modulates airway epithelium susceptibility to death receptor-mediated apoptosis. Am J Physiol Lung Cell Mol Physiol 2006;290:L433-41.

29. Prasad AS. Zinc in human health: effect of zinc on immune cells. Mol Med 2008;14:353-7.

30. Coles CL, Sherchand JB, Khatry SK, et al. Zinc modifies the association between nasopharyngeal Streptococcus pneumoniae carriage and risk of acute lower respiratory infection among young children in rural Nepal. J Nutr 2008;138:2462-7. 